\title{
THE CHANGING CONCEPTS IN THE RETENTION OF MAXILLOFACIAL PROSTHESIS FROM PAST TO PRESENT- A REVIEW
}

\author{
Hema Kanathila1, Ashwin Pangi ${ }^{2}$
}

${ }^{1}$ Reader, Department of Prosthodontics, KAHE's KLE VK Institute of Dental Sciences.

${ }^{2}$ Reader, Department of Prosthodontics, Vasantdada Patil Dental College, Sangli.

ABSTRACT

\section{BACKGROUND}

Defects in the maxillofacial region can be congenital or acquired, both always makes an individual depressed, hence requires rehabilitation. Maxillofacial prosthesis helps in replacing the congenitally missing or lost body part due to trauma. From the past, the fitting and attachment of the prosthesis to the patient continues to be a great challenging factor, which needs improvement. Margins are difficult to mask even with the best materials. Large prostheses of most of the materials are too heavy and poorly retained. Retention plays a very important role in retaining the prostheses and thereby increasing patient's comfort and confidence. Using ideal retentive aids along with the aesthetic, functional and economic factors helps in achieving successful outcome.

\section{KEYWORDS}

Retention, Maxillofacial Prosthesis, Auricular Prosthesis, Ocular Prosthesis, Intraoral Prosthesis.

HOW TO CITE THIS ARTICLE: Kanathila H, Pangi A. The changing concepts in the retention of maxillofacial prosthesis from past to present- a review. J. Evolution Med. Dent. Sci. 2017;6(84):5879-5883, DOI: 10.14260/jemds/2017/1277

\begin{abstract}
BACKGROUND
Prosthodontist has a major role in rehabilitation of maxillofacial defects. Prosthetic reconstruction of a defect is highly challenging for a prosthodontist and it depends on various factors such as size, site, aetiology, extension, age, patient's comfort, satisfaction and cost factor. The materials used for rehabilitation have travelled a long way from wood to polymers and the retentive aids used from metal bands to implants. Facial defects can be congenital or acquired. Acquired defects are mostly due to trauma or as a result from treatment of neoplasms. A surgeon and prosthodontist with their knowledge, skill and experience have to decide the best mode of retention possible for a maxillofacial prosthesis. Thorough evaluation of the defect before and during surgery will help to modify the irregular defects to aid in retention in some cases. In cases of large maxillofacial defects, movement of prosthesis poses a real challenge. A careful treatment planning can give a better quality of maxillofacial prostheses, which improves the patient's quality of life. A team effort is essential for the effective treatment of patients with maxillofacial problems.
\end{abstract}

\section{The Primary Objectives of Maxillofacial Rehabilitation} are1

1. Restoration of aesthetics- By rehabilitating maxillofacial defect patients, a prosthodontist restores the orofacial appearance, thereby enhancing the cosmetic acceptability of the patient. An aesthetically appealing facial prosthesis should reproduce the form, size, position, texture and colour of the lost tissue.

'Financial or Other Competing Interest': None.

Submission 08-09-2017, Peer Review 07-10-2017,

Acceptance 12-10-2017, Published 19-10-2017.

Corresponding Author:

Dr. Hema Kanathila,

Reader, Department of Prosthodontics,

KAHE'S KLE VK Institute of Dental Sciences,

KLE Academy of Higher Education and Research University,

Belagavi.

E-mail: hemak_19@yahoo.com

DOI: $10.14260 /$ jemds $/ 2017 / 1277$
2. Restoration of function- Functional disparity due to loss of orofacial structures causes health problems and psychological disorders. Hence, rehabilitation of maxillofacial defects helps in restoring functions of mastication, deglutition and speech.

3. Psychological benefit- The impossibility of leading a normal social life results in serious psychological disorders. Thus, rehabilitating the facial deformity helps in achieving the facial symmetry and thereby allowing those individuals to restore their active role in society.

4. Therapeutic effect- After surgical procedures in trauma and carcinoma cases, maxillofacial prosthetic aids help by acting as carriers for medicinal applications.

5. Preservation of tissues- The main goal of a prosthodontist is the perpetual preservation of what is present. Maintaining the tooth, bone and surrounding orofacial structures is considered to achieve success in maxillofacial prosthetic rehabilitation cases.

\section{Retention}

According to GPT-9 (The Glossary of Prosthodontic Terms), retention is that quality inherent in the dental prosthesis acting to resist the forces of dislodgement along the path of placement.

Any Prosthesis serves its Purpose only when it is Retentive. There are 4 different ways by which Anchorage can be achieved in Maxillofacial Prosthesis, they include-

1. Anatomic retention.

2. Mechanical retention.

3. Chemical retention.

4. Surgical retention. ${ }^{2}$

\section{Anatomic Retention}

Anatomic undercut areas can always be created by planning before and after surgery as a mode of retention for maxillofacial prosthesis. Anatomical retention is obtained by already existing anatomical structures just as in the case of 
undercut area in ocular defects. ${ }^{2}$ Anatomic retention can be either intraoral or extraoral.

\section{Intraoral Retention}

Intraoral retention is achieved by the use of hard and soft tissues. It can be from teeth, mucosal and bony tissues. Anatomic undercuts are found in the palatal area, cheek, retromolar area, remaining teeth, alveolar ridge, septum and anterior nasal aperture. ${ }^{3}$ Large alveolar ridge and high arched palate provides greater retention than flat ridges and palate. Intraoral retentive aids are usually considered comfortable for the patient for easy removal and for the examination of the surgical site by the dentist in order to check for recurrence of tumour.

\section{Extraoral Retention}

The movement of the prosthesis has the possibility of creating stress on the abutment teeth, leading to the loss of the tooth. Therefore, in such cases an additional retention can be used. Extraoral retention can be achieved from hard and soft tissues of maxillofacial and neck region. Deep undercuts create difficulties in insertion and removal of prosthesis. Soft tissues create problem due to their mobility and lesser resistance to displacement when a force is applied. ${ }^{3}$ Soft tissue undercuts are usually in the maxillary sinus, nasal cavity and orbital regions. The advantages of prosthesis used in these areas are that they are cost effective, aesthetic and easy to fabricate.

\section{Chemical Retention}

Chemical retention is provided by Adhesives. According to GPT-9, maxillofacial prosthetic adhesive is "a material used to adhere external prosthesis to the skin and associated structures around the periphery of an external anatomic defect." Adhesives are considered as the most popular retentive aid in maxillofacial prosthesis retention. The selection of an adhesive is based on certain criteria. They include-

1. Bond strength of the adhesive to the prosthetic material and recipient tissues.

2. Biocompatibility.

3. Prosthesis design.

4. Type and quality of patient's skin.

5. Composition and viscosity.

6. Handling, storage and shelf life. ${ }^{3}$

These are available as acrylic or silicone based adhesives, latex, spirit gum or water based adhesives.,3 Acrylic resin adhesives consists of acrylic resin dispersed in a water solvent. It leaves a rubber-like substance which when evaporated provides the adhesiveness. Silicone adhesives are a form of room-temperature vulcanising silicones, which are dissolved in a solvent. This solvent evaporates and the resulting tacky adhesive helps in retention. Pressuresensitive tapes consists of a backing strip composed of cloth, paper, foil or a laminate strip coated with a pressure sensitive adhesive which provides retention. Examples of adhesives are Pros-Aide adhesive, Epithane-3 adhesive, 3M bifaceis, Hollister Medical Adhesive.

A study conducted by Kiat-Amnuay et al, evaluated time and reapplication effects of adhesive retention of maxillofacial prostheses. According to this study, bond strengths decreased during the course of the day because of perspiration and normal body motion. It was noted that the application of a second coat of adhesive after an interval of 4 to 8 hours enhanced the retention of the silicone elastomeric strips. $^{5}$

\section{Advantages}

Adhesives are cost effective and easy to manipulate and apply. Maxillofacial defect patients who are not willing for implant surgical procedures consider adhesives as a retentive aid.

\section{Disadvantages}

Certain adhesives require solvents to clean after removal of prosthesis. It provides an unreliable retention. Its degradation to the prosthetic material adds to its disadvantages apart from irritation, perspiration and movement that compromises the bond. In some patients, it may cause allergic reactions. ${ }^{3}$

\section{Mechanical Retention}

Mechanical anchorage includes-

1. Magnets.

2. Eye glasses and frames.

3. Extension from denture.

4. Precision attachments.

5. Elastic and non-elastic straps.

\section{Magnets}

Magnets gained popularity in the field of maxillofacial prosthesis due to their small size and strong attractive forces. ${ }^{6}$ According to the size of the defect, most appropriate size of magnet can be chosen. Magnets are used as retentive aid for sectional dentures, hemi-maxillectomy, obturators, complete dentures or extensively atrophied ridges. They are said to provide the best retention and stabilisation for maxillofacial prosthesis. Magnets as a retentive aid help in the attachment of implant to the prosthesis. Magnetic attachments on the teeth and on implants are used to improve stability, support and retention of prostheses.

Starting from the past, many researchers have studied on different magnetic systems and used magnet as retentive aid in maxillofacial prosthesis cases. Usually, in large maxillary defects, a sectional prosthesis is considered as a treatment option. In this case, two sections are united using two magnetic pairs. Robinson used horseshoe magnets for the retention of an upper denture and obturator in a total maxillectomy case. He described a method of constructing a two-section intraoral prosthesis using attracting magnets as positive locking devices. ${ }^{7}$ Matsamura et al described fabrication procedure of a removable sectional denture connected by a cobalt-samarium magnetic retention system for a patient with severe maxillary defect. ${ }^{8}$

Mainly two types of alloys are used for the manufacture of small dental magnets. They are cobalt-samarium and ironneodymium and boron. They have high attractive forces in very small sizes, but have low corrosion resistance. ${ }^{9}$ Tsutsui $\mathrm{H}$ et al found Samarium-Cobalt magnet to be superior in magnetic properties to other magnets. ${ }^{10}$ Grant GT et al (2001) described a procedure for the fabrication of an extraoral prosthesis with an acrylic resin substructure that retains a magnet sealed from the environment by a polyurethane 
liner. ${ }^{11}$ Yoshida $\mathrm{K}$ et al (2008) treated two female patients with orbital defects using custom-made retentive components of an individual magnet for an Epitec System orbital prosthesis. ${ }^{12}$

Fe-Pt dental magnetic attachments are clinically useful for retention of maxillofacial prostheses due to their excellent attractive force. Fe-Pt magnetic attachment system (magnet and keeper) can be cast in a dental casting machine. Hence, any size or shape of castable magnetic attachment can be fabricated for maxillofacial prostheses. ${ }^{13}$ A case was presented, where a coin shaped magnet was used for retaining a facial prosthesis combined with complete dentures in a patient who had undergone the removal of left maxilla, the palatine bone, a part of the zygomatic bone and the complete left orbit.14 Voigt A et al determined which combination of differently designed magnetic abutments provided the best retention for an auricular prosthesis. The highest withdrawal force was found in the combination of one telescopic magnet and two large spherical magnets. ${ }^{15}$

Magnets are used in both mandibular and maxillary implant-supported, full-arch bar, fixed-detachable prosthesis in the fabrication of sectional intraoral maxillofacial prostheses. Retentive forces by these magnetic attachments are limited against lateral masticatory forces. ${ }^{13}$ In such cases, additional retention should be considered. Retention of the prosthesis in large maxillofacial defects resulting from cancer resection is challenging due to two reasons- size and weight. In this condition, retention can be obtained by adhesives, resilient attachments, implants or it can be united to an obturator by magnets. Federick DR (1976) put forward a technique for the fabrication of a sectional interim maxillary obturator with retention augmented by a magnet. ${ }^{16}$ Mahnaz Hatami et al constructed a complete denture with obturator as well as facial prosthesis, which can be attached to the obturator with cobalt samarium magnets in a patient who was affected by a fungal infection and resection of hard palate, nasal septum, maxillary sinus and orbital contents was done. ${ }^{17}$

Magnetically retained overdentures require less maintenance and relatively inexpensive. Majage et al reported a case of hemi-mandibulectomy where prosthodontic rehabilitation was done with magnet retained overdenture. ${ }^{18}$ Seema Pattanaik et al described rehabilitation of a patient with subtotal maxillectomy and enucleated eye by intraoral prosthesis and extraoral orbital prosthesis retained with magnets. ${ }^{19}$ Banerjee et al described rehabilitation of patient with midfacial defect with two piece prosthesis by the use of magnets. ${ }^{20} \mathrm{~A}$ case report of partial nasal defect caused by trauma, rehabilitated with a magnetic nasal prosthesis made with silicone elastomers using mechanical and anatomical retentive aids was mentioned by Nagaraj E et al. ${ }^{21}$

Advantages of using magnets are ease of placement, automatic reseating, easy replacement, small size with strong attractive forces can be placed within the prostheses and ease of cleaning.

\section{Eyeglasses}

They are used in ocular, nasal and auricular prosthesis. Muddugangadhar et al treated an orbital defect patient by attaching the ocular prosthesis with spectacles. It also helps in masking the borders of the prosthesis. ${ }^{22}$ Advantages of using eye glass as retentive aid can be mentioned as easy, economic and practical.

\section{Extension from Denture}

Most primitive type of retentive aids namely cast clasps, retentive clips and acrylic buttons are still being used as they are the most economical amongst the others.

\section{Precision Attachments}

Bar clips are most commonly used precision attachment that connects the prostheses and implant and between different parts of prosthesis. Telescopic crowns and extracoronal ball attachments are used to increase and improve retentive force in maxillofacial prosthesis cases.

\section{Elastic and Non-Elastic Straps}

They are used with extraoral prosthesis. Head bands are used in cases of auricular prosthesis. Non-elastic straps are used along with buckles to make it adjustable. It requires a head cap to gain anchorage from. Orthodontic headgear assemblies like head cap and adjustable strap extension are very useful for extensive maxillofacial prosthesis. ${ }^{4}$

\section{Surgical Retention}

Titanium implants can be placed for fixation of prosthetic ears, nose and eyes. In facial deformities, zygomatic implants, orbital, auricular and nasal implants are the extraoral implants placed. They provide most reliable form of retention for maxillofacial prosthesis. It also enhances function of prosthesis and good marginal fit makes the margins less obvious. Placement of osseointegrated implants has a great effect on the function of facial prosthesis in a matter of retention, stability and support.4,23,24,25,26,27 Most commonly used are cylindrical or tapered root form titanium implants. They are mechanically anchored to bone. Marius Implant Bridge is a prosthetic rehabilitation for the completely edentulous upper jaw with moderate-to-severe resorption cases. ${ }^{28}$

The most ideal location for implants in edentulous total maxillectomy patients is residual premaxilla. Zygomatic implants are also used in the treatment of maxillary defects secondary to trauma, tumour resection or any congenital defects. For nasal prosthesis, ideal site is maxilla region and anterior floor of nose with tissue bar and clip design. Paolo Cariati et al rehabilitated a nasal defect caused by squamous cell carcinoma using three implants. He concluded that effective reconstruction, optimum aesthetic would be achieved and positive results in psychosocial well-being of patient were found. ${ }^{29}$

The ability to recreate the normal anatomy and the reaction of the orbital soft tissue to the biomaterial used in reconstruction are two major factors that are important while reconstructing the internal orbit. Supraorbital rim or lateral rim of orbit is the preferred site for ocular prosthesis.

Nilgun A et al fabricated an auricular prosthesis for a patient who lost left and right external ear in an electric burn. In this case report, extraoral implants and bar and clip retention for the proper connection of auricular prosthesis were used. ${ }^{30}$ Aydin $C$ et al (2008) found implant success rate to be $100 \%$ for silicone auricular prosthesis. ${ }^{31}$

Hyung In Yoon et al, rehabilitated a patient with implant supported fixed prosthesis using computer aided design and 
computer aided dental technology for a patient with mandibulectomy. ${ }^{32}$

PEEK (Polyetherether Ketone) is a polymer which has become recently familiar due to their biocompatibility, strength, stiffness and durability. ${ }^{33}$ Owing to its mechanical and physical properties being similar to bone and dentin, PEEK can be used for a number of applications in dentistry. ${ }^{34}$ PEEK exhibits excellent biocompatibility and stability when exposed to body fluids. Few of the cranial, frontal, ocular, maxillary and mandibular defects are being reconstructed by 3D printing using PEEK (Polyetherether ketone). PEEK implants can be machined to many organic shapes and fixated to the adjacent bone standard screws and plates. ${ }^{35}$

Kim et al described a series of 4 patients, whose defects were reconstructed using customised PEEK implants. The patients showed excellent postoperative aesthetic and functional results without complications such as infections or extrusions. ${ }^{33}$

\section{CONCLUSION}

To reduce the burden of physical and psychological trauma and for the well-being of the patient, the replacement of facial defect becomes the responsibility of the maxillofacial prosthodontist. The aesthetics achieved after complete treatment depends on the amount of tissue removed, good contour and merging of the margins and minimal sagging due to the weight of the prosthesis. The advantage of maxillofacial prostheses is that it requires minimal or no surgery, as it restores the aesthetics and function in a near natural appearance. Retention is a key factor in maxillofacial prosthesis. The need for professional evaluation on a periodic basis should be encouraged to determine the adaptability of prosthesis to soft tissues, stability, retention, function and aesthetics.

\section{REFERENCES}

[1] Deba K. Oral \& Maxillofacial Prosthetics-I: Objectives \& History. Heal Talk 2012;4(5).

[2] Karthikeyan I, Khatree M, Gaddale R, et al. A review on prosthetic rehabilitation of maxillofacial region. Journal of Scientific Dentistry 2016;6(1):6-12.

[3] Gurjar R, Kumar SMV, Rao H, et al. Retentive aids in maxillofacial prosthodontics - a review. IJCD 2011:2(3);84-8.

[4] Yeshwante B, Patil SJ, Baig N. Retentive aids used in maxillofacial prosthesis. IJCDS 2014;5(2):12-20.

[5] Kiat-Amnuay S, Gettleman L, Khan Z, et al. Effect of adhesive retention of maxillofacial prostheses. Part 2: time and reapplication effects. J Prosthet Dent 2001;85(5):438-41.

[6] Bhat VS, Shenoy KK, Premkumar P, et al. Magnets in dentistry. AMHS 2013;1(1):73-9.

[7] Robinson JE. Magnets for the retention of a sectional intraoral prosthesis: a case history. J Prosthet Dent 1963;13(6):1167-71.

[8] Matsumura H, Kawasaki K. Magnetically connected removable sectional denture for a maxillary defect with severe undercut: a clinical report. The Journal of Prosthetic Dentistry 2000;84(1):22-6.

[9] Highton R, Caputo AA, Pezzoli M, et al. Retentive characteristics different magnetic systems for dental applications. J Prosthet Dent 1986;56(1):104-6.
[10] Tsutsui H, Kinouchi Y, Sasaki H, et al. Studies on the Sm-Co magnet as a dental material. J Dent Res 1979;58(6):1597-606.

[11] Grant GT, Taft RM, Wheeler ST. Practical application of polyurethane and Velcro in maxillofacial prosthetics. J Prosthet Dent 2001;85(3):281-3.

[12] Yoshida K, Takagi A, Tsuboi Y, et al. Modified hygienic Epitec System abutment for magnetic retention of orbital prostheses. J Prosthodont 2008;17(3):219-22.

[13] Lakshmi NS, Bharathi M, Sujitha K, et al. Magnets and their role in prosthodontics. International Journal of Oral Health and Medical Research 2016;3(1):155-7.

[14] Javid N. The use of magnets in maxillofacial prosthesis. The Journal of Prosthetic Dentistry 1971;25(3):334-41.

[15] Voigt A, Christ S, Klein M. Experimental analysis of retention forces of different magnetic devices for bone anchored auricular facial prostheses. Int J Oral Maxillofac Surg 2008;37(7):664-8.

[16] Federick DR. A magnetically retained interim maxillary obturator. The Journal of Prosthetic Dentistry 1976;36(6):671-5.

[17] Hatami M, Badrian H, Samanipoor S, et al. Magnetretained facial prosthesis combined with maxillary obturator. Case Reports in Dentistry Article ID 406410, 2013;2013: p. 5.

[18] Basavakumar M, Neeraj C, Ramesh C, et al. Prosthodontic rehabilitation of hemi mandibulectomy patient with magnet retained overdenture - a case report. Int Journal of Clinical Dental Science 2010;1(1):30-2.

[19] Pattanaik S, Wadkar AP. Rehabilitation of a patient with intra oral prosthesis and an extra oral orbital prosthesis retained with magnets. J Indian Prosthodont Soc 2012;12(1):45-50.

[20] Banerjee S, Kumar S, Bera A, et al. Magnet retained intraoral-extra oral combination prosthesis: a case report. The J Adv Prosthodont 2012;4(4):235-8.

[21] Nagaraj E, Shetty M, Krishna PD. Definitive magnetic nasal prosthesis for partial nasal defect. Indian J Dent Res 2011;22(4):597-9.

[22] Muddugangadhar BC, Sonika R, Chheda PS, et al. Rehabilitation of an orbital defect: a simplified technique. J Int Oral Health 2015;7(7):121-3.

[23] Kantola R. Use of fibre-reinforced composite framework and thermochromic pigment in facial prosthesis. Turun Yliopisto University of Turku. Turku 2014: p. 13.

[24] Parel SM, Tjellström A. The United States and Swedish experience with osseointegration and facial prostheses. Int $\mathrm{J}$ Oral Maxillofac Implants 1991;6(1):75-9.

[25] Watson RM, Coward TJ, Forman GH. Results of treatment of 20 patients with implants-retained auricular prostheses. Int J Oral Maxillofac Implants 1995;10(4):445-9.

[26] Arcuri MR, Rubenstein JT. Facial implants. Dent Clin North Am 1998;42(1):161-75.

[27] Hooper SM, Westcott T, Evans PLL, et al. Implantsupported facial prostheses provided by a maxillofacial unit in a U.K. regional hospital: longevity and patient options. J Prosthodont 2005;14:32-7. 
[28] Fortin Y, Sullivan RM, Rangert BR. The Marius implant bridge: surgical and prosthetic rehabilitation for the completely edentulous upper jaw with moderate to severe resorption: a 5-year retrospective clinical study. Clin Implant Dent Relat Res 2002;4(2):69-77.

[29] Cariati P, Solis JF, De Perceval MP, et al. Reconstruction of mid-facial defects using facial prostheses supported by dental implants. J Aesthet \& Reconstr Surg 2016;2(2):14.

[30] Aydin C, Karakoca S, Yilmaz H, et al. Implant- retained auricular prostheses: an assessment of implant success and prosthetic complications. Int J Prosthodont 2008;21(3)241-4.

[31] Yoon HI, Han JS. Prosthetic rehabilitation with an implant-supported fixed prosthesis using computeraided design and computer-aided manufacturing dental technology for a patient with a mandibulectomy: a clinical report. Journal of Prosthetic Dentistry 2016;115(2):133-6.
[32] Ozturk NA, Usumez A, Tosun Z, et al. Implant-retained auricular prosthesis: a case report. European Journal of Dentistry 2010;4(1):71-4.

[33] Kim MM. Use of customized polyetheretheretone (PEE) implants in the reconstruction of comple maxillofacial defects. Arch Facial Plas Surg 2009;11(1):53-7.

[34] Najeeb S, Zafar MS, Khurshid Z, et al. Applications of polyetheretherketone (PEEK) in oral implantology and prosthodontics. Journal of Prosthodontic Research 2016;60(1):12-9.

[35] Herford AS, Miller M, Lauritano F, et al. The use of virtual surgical planning and navigation in the treatment of orbital trauma. Chinese Journal of Traumatology 2017;20(1):9-13. 